\title{
A PRELIMINARY INVESTIGATION ON COMPARISON AND TRANSFORMATION OF SENTINEL-2 MSI AND LANDSAT 8 OLI
}

\author{
Feng Chen ${ }^{1 *}$, Shenlong Lou ${ }^{1}$, Qiancong Fan ${ }^{1}$, Jonathan Li ${ }^{1,2 *}$, Cheng Wang ${ }^{1}$, Martin Claverie ${ }^{3}$ \\ ${ }^{1}$ Fujian Key Laboratory of Sensing and Computing for Smart Cities, Xiamen University, Xiamen, China \\ ${ }^{2}$ Department of Geography and Environmental Management, University of Waterloo, Waterloo, Canada \\ ${ }^{3}$ University of Maryland, Department of Geographical Sciences, College Park, MD, USA
}

Commission III, WG III/6

KEY WORDS: Sentinel-2, Landsat, Spectral response function (SRF), Cross-calibration, Transformation, Consistency, Uncertainty

\begin{abstract}
:
Timely and accurate earth observation with short revisit interval is usually necessary, especially for emergency response. Currently, several new generation sensors provided with similar channel characteristics have been operated onboard different satellite platforms, including Sentinel-2 and Landsat 8. Joint use of the observations by different sensors offers an opportunity to meet the demands for emergency requirements. For example, through the combination of Landsat and Sentinel-2 data, the land can be observed every 2-3 days at medium spatial resolution. However, differences are expected in radiometric values (e.g., channel reflectance) of the corresponding channels between two sensors. Spectral response function (SRF) is taken as an important aspect of sensor settings. Accordingly, between-sensor differences due to SRFs variation need to be quantified and compensated. The comparison of SRFs shows difference (more or less) in channel settings between Sentinel-2 Multi-Spectral Instrument (MSI) and Landsat 8 Operational Land Imager (OLI). Effect of the difference in SRF on corresponding values between MSI and OLI was investigated, mainly in terms of channel reflectance and several derived spectral indices. Spectra samples from ASTER Spectral Library Version 2.0 and Hyperion data archives were used in obtaining channel reflectance simulation of MSI and OLI. Preliminary results show that MSI and OLI are well comparable in several channels with small relative discrepancy $(<5 \%)$, including the Costal Aerosol channel, a NIR (855-875 $\mathrm{nm}$ ) channel, the SWIR channels, and the Cirrus channel. Meanwhile, for channels covering Blue, Green, Red, and NIR (785-900 $\mathrm{nm}$ ), the between-sensor differences are significantly presented. Compared with the difference in reflectance of each individual channel, the difference in derived spectral index is more significant. In addition, effectiveness of linear transformation model is not ensured when the target belongs to another spectra collection. If an improper transformation model is selected, the between-sensor discrepancy will even largely increase. In conclusion, improvement in between-sensor consistency is possibly a challenge, through linear transformation based on model (s) generated from other spectra collections.
\end{abstract}

\section{INTRODUCTION}

For some special event (i.e., monitoring and assessment for emergency risk), meaningful information available to users is necessarily required. This service relies on timely and accurate earth observation with short revisit interval. However, the needs are impossible or difficult to meet through an individual sensor or platform. Currently, several new generation sensors with similar channel characteristics have been operated onboard different satellites, including Sentinel-2 and Landsat 8. Joint use of the observations obtained from different sensors may offer an opportunity to meet the demands for emergency requirements. Two Sentinel-2 satellites (named Sentinel-2A and Sentinel-2B) with identically planned sensor-the Multi-Spectral Instrument (MSI), although differences are observed more or less (Chen et al., 2018), have been operated currently. By the way, two additional satellites in the Sentinel-2 series, are scheduled to launch starting in 2021 (http://spacenews.com/thales-aleniaspace-signs-contract-to-build-2-more-sentinel-satellites-foresa). Sentinel-2A was launched on 23 June 2015, while Sentinel-2B satellite was launched on 7 March 2017. When two Sentinel-2 satellites are operated simultaneously, the mission's revisit time is about 5 days, while about 10 days with one satellite operated. The MSI imagery consists of 13 spectral channels, from the visible and the near-infrared (VNIR) to the shortwave infrared (SWIR) at suitable spatial resolutions. Specifically, there are 4 channels at 10 meters, 6 channels at 20 meters, and 3 channels at 60 meters (Figure 1 and Table 1). Meanwhile, Landsat 8, launched on 11 February 2013, is the newest member of the Landsat program. There are two instruments onboard Landsat 8, including the Operational Land Imager (OLI) and Thermal Infrared Sensor (TIRS). The OLI imagery consists of 9 spectral channels, of which 8 multispectral channels are provided with a spatial resolution of 30 meters and a panchromatic channel with a spatial resolution of 15 meters (Figure 1 and Table 1). By the way, the Landsat program has been collecting space-based imagery with moderate spatial resolution of the Earth's surface since the launch of its first member (Landsat 1) in 1972. Furthermore, free accessibility of the observations from Sentinel-2 MSI and Landsat 8 OLI shows obvious advantage in the joint use of these data in terms of application, data quality and spatial resolution. Accordingly, the combination of Landsat and Sentinel-2 data offers a unique opportunity to observe land every 2-3 days at medium spatial resolution (Claverie et al.,

\footnotetext{
*Feng Chen (chenfeng@xmu.edu.cn; chenfeng105@gmail.com);

Jonathon Li (junli@uwaterloo.ca)
} 
2017). However, the joint use of observations from different sensors poses challenges, mainly due to the differences in orbital, spatial, and spectral configuration (Mandanici and Bitelli, 2016). Consequently, some differences are expected in the recorded radiometric values (e.g., channel reflectance) of the corresponding channels between different sensors (i.e., MSI and OLI). Spectral response function (SRF) was taken as an important aspect of sensor settings, as discussed previously (Chander et al., 2013; Chen et al., 2016; Chen et al., 2017). Previous findings suggest that the effect of differences due to SRFs of the sensors need to be quantified and compensated to avoid large uncertainties in cross-calibration results (Chander et al., 2013).

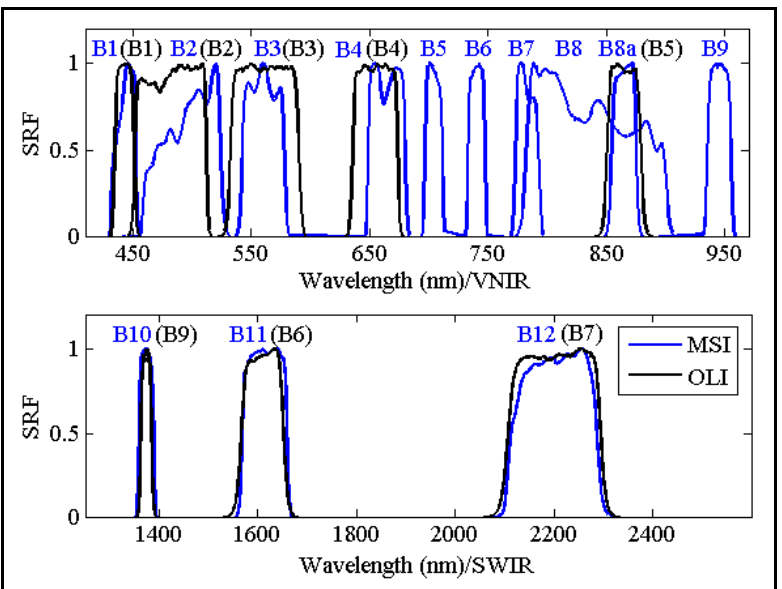

Figure 1. Spectral response functions (SRFs) of Sentinel-2A MSI channels (blue line) and Landsat 8 OLI (black line) over the visible and near infrared (VNIR) regions (up) and the shortwave infrared (SWIR) regions (below).

\begin{tabular}{|c|c|c|c|c|c|}
\hline \multicolumn{3}{|c|}{ Landsat 8 OLI } & \multicolumn{3}{c|}{ Sentinel-2 MSI } \\
\hline Band & $\begin{array}{c}\text { Wavelength } \\
(\mathrm{nm})\end{array}$ & $\begin{array}{c}\text { Res\# } \\
(\mathrm{m})\end{array}$ & Band & $\begin{array}{c}\text { Wavelength } \\
(\mathrm{nm})\end{array}$ & $\begin{array}{c}\text { Res } \\
(\mathrm{m})\end{array}$ \\
\hline 1 CA* & $433-453$ & 30 & 1 CA & $433-453$ & 60 \\
\hline 2 Blue & $450-515$ & 30 & 2 Blue & $458-523$ & 10 \\
\hline 3 Green & $525-600$ & 30 & 3 Green & $543-578$ & 10 \\
\hline 4 Red & $630-680$ & 30 & 4 Red & $650-680$ & 10 \\
\hline 5 NIR & $845-885$ & 30 & 8 NIR & $785-900$ & 10 \\
\cline { 4 - 7 } & & & 8a NIR & $855-875$ & 20 \\
\hline 6 SWIR1 & $1560-1660$ & 30 & 11 SWIR1 & $1565-1655$ & 20 \\
\hline 7 SWIR2 & $2100-2300$ & 30 & 12 SWIR2 & $2100-2280$ & 20 \\
\hline 9 Cirrus & $1360-1390$ & 30 & 10 Cirrus & $1360-1390$ & 60 \\
\hline
\end{tabular}

Table 1. Corresponding spectral channels and resolutions of OLI and MSI (*CA: coastal aerosol; \#Res: Resolution)

Comparison between MSI and OLI is to provide basis for joint use of different observations. As shown in Figure 1, differences in SRFs are significant over several channels (i.e., in VNIR region), while differences of SRFs for other channels are minor (Figure 1). Effect of the difference in SRF on corresponding values between MSI and OLI was investigated, including channel reflectance and derived spectral index. Investigations were mainly based on spectra simulation. Valid spectra samples from ASTER Spectral Library Version 2.0 and Hyperion data archives were separately used to obtain the channel reflectance. Descriptions on channel reflectance calculation and the spectra collections are detailed in Section 2. Major findings of the preliminary comparison are mainly shown in Section 3 . Sentinel-2A MSI was mainly discussed in this paper, whereas, actually Sentinel-2B MSI is different with Sentinel-2A MSI more or less as shown in other investigation (Chen et al., 2018).

\section{METHODS AND DATA}

\subsection{Channel Reflectance}

Channel reflectance as an effective value over a specific channel is obtained through Eq. (1).

$$
\operatorname{Ref}_{\mathrm{Bi}}=\frac{\int_{\lambda s}^{\lambda_{\mathrm{E}}} \operatorname{SRF}^{\mathrm{Bi}}(\lambda) \operatorname{Ref}(\lambda) \mathrm{d} \lambda}{\int_{\lambda_{\mathrm{s}}}^{\lambda_{\mathrm{E}}} \operatorname{SRF}^{\mathrm{Bi}}(\lambda) \mathrm{d} \lambda}
$$

where $\operatorname{SRF}^{\mathrm{Bi}}(\lambda)$ is the SRF of a specific channel $\mathrm{Bi}$, while $\lambda_{\mathrm{S}}$ and $\lambda_{\mathrm{E}}$ are the start wavelength and the end wavelength of the channel spectral range. $\operatorname{Ref}(\lambda)$ is the reflectance at a specific wavelength $\lambda$.

The corresponding spectral channels of OLI and MSI are presented in Table 1. Comparisons of these corresponding channels were based on channel reflectance. Specific processes were adopted to calculate channel reflectance through Eq. (1), for ASTER spectra collection and Hyperion spectra collection respectively, which were detailed in reference (Chen et al., 2018).

\subsection{Spectral Index}

Comparison of spectral index was also discussed to investigate the effects of difference in channel SRFs. The spectral index is widely used as a practical way to delineate surface cover or status. In this paper, two spectral indices were discussed, including Normalized Difference Vegetation Index (NDVI, Eq. (2)) and Normalized Difference Built-up Index (NDBI, Eq. (3)) (Zha et al., 2003). Both NDVI and NDBI have been used as measures to characterize landscape and to model urban thermal environment (Xiong et al., 2012).

$$
\begin{gathered}
\mathrm{NDVI}=\frac{\left(\operatorname{Ref}_{n i r}-\operatorname{Ref}_{\text {red }}\right)}{\left(\operatorname{Ref}_{n i r}+\operatorname{Ref}_{r e d}\right)} \\
\mathrm{NDBI}=\frac{\left(\operatorname{Ref}_{\text {swir }}-\operatorname{Ref}_{\text {nir }}\right)}{\left(\operatorname{Ref}_{\text {swir }}+\operatorname{Ref}_{\text {nir }}\right)}
\end{gathered}
$$

where $\operatorname{Ref}_{\text {red }}, \operatorname{Ref}_{n i r}, \operatorname{Ref}_{\text {swir }}$ are red channel, near infrared channel, and short wave infrared channel, respectively. Descriptions of these channels are presented in Figure 1 and Table 1.

MSI has two channels located within NIR range (labelled as "8" and "8a"), while OLI has one NIR channel (Figure 1 and Table 1). Regarding to MSI, the selection of NIR channel for both NDVI and NDBI calculation is to be discussed, although the channel " 8 " is recommended for NDVI calculation according to the descriptions shown at the official web (https://sentinel.esa.int/web/sentinel/technicalguides/sentinel-2msi/level-2a/algorithm). Meanwhile, the channel "SWIR1" was used for NDBI calculation.

\subsection{Difference Measurement}

To show generally overall measures of the difference in channel reflectance between corresponding channels of MSI and OLI, three indicators were used, including the mean difference (MD, Eq. (4)), the root mean square deviation (RMSD, Eq. (5)), and 
the mean relative difference (MRD, Eq. (7)). The indicators were used previously (Roy et al., 2016). Meanwhile, relative difference (RD) defined as Eq. (6) measures individual relative difference (j) in corresponding variable (i). To measure the average relative difference in spectral index, the median relative difference (MdRD, Eq. (8)) was used.

$$
\begin{gathered}
\mathrm{MD}_{\mathrm{i}}=\operatorname{mean}\left(\operatorname{Var}_{\mathrm{i}}^{\mathrm{MSI}}-\operatorname{Var}_{\mathrm{i}}^{\mathrm{OLI}}\right) \\
\operatorname{RMSD}_{\mathrm{i}}=\operatorname{sqrt}\left(\operatorname{mean}\left(\left(\operatorname{Var}_{\mathrm{i}}^{\mathrm{MSI}}-\operatorname{Var}_{\mathrm{i}}^{\mathrm{OLI}}\right)^{2}\right)\right) \\
\mathrm{RD}_{\mathrm{ij}}=2 \times \frac{\left(\operatorname{Var}_{\mathrm{ij}}^{\mathrm{MSI}}-\operatorname{Var}_{\mathrm{ij}}^{\mathrm{OLI}}\right)}{\left(\operatorname{Var}_{\mathrm{ij}}^{\mathrm{MSI}}+\operatorname{Var}_{\mathrm{ij}}^{\mathrm{OLI}}\right)} \times 100 \\
\operatorname{MRD}_{\mathrm{i}}=\operatorname{mean}\left(\mathrm{RD}_{\mathrm{ij}}\right) \\
\operatorname{MdRD}_{\mathrm{i}}=\operatorname{median}\left(\mathrm{RD}_{\mathrm{ij}}\right)
\end{gathered}
$$

where $\operatorname{Var}_{\mathrm{ij}}^{\mathrm{OLI}}$ and $\operatorname{Var}_{\mathrm{ij}}^{\mathrm{MSI}}$ are corresponding values of $\mathrm{j}^{\text {th }}$ samples in variable (i) for Sentinel-2 MSI and Landsat 8 OLI respectively (i.e., of individual channel reflectance or spectral index), while mean( ), sqrt( ), and median( ) are procedures for obtaining the mean value, square root value, and median value, respectively.

\subsection{Transformation Model}

Linear model has been used for cross-sensor transformation, as a practical means to ensure comparability between different sensors. Accordingly, the linear model used for transforming MSI observation to corresponding value of OLI is investigated (Eq. (9)).

$$
\operatorname{Var}_{\mathrm{i}}^{\mathrm{OLI}}=\text { Slope } \times \operatorname{Var}_{\mathrm{i}}^{\mathrm{MSI}}+\text { Offset }
$$

where "Slope" and "Offset" are parameters in the linear transformation model.

In this paper, both improvements and uncertainty associated with a linear transformation model were discussed. Uncertainty of the linear model was presented by the distribution of two modelling parameters (i.e., "Slope" and "Offset"). The improvements in variable consistency between two sensors due to transformation model were shown through the comparison of original between-sensor difference and model prediction error. Discussions on both issues were based on statistics of K-fold cross-validation $(K=5)$ with 10,000 simulations. That is, for each validation case, $80 \%$ spectra pairs are selected for training and the rest $20 \%$ are for testing, mean prediction error and mean difference (the original, before transformation) are measured and compared accordingly.

\subsection{Spectra Data}

Effect of the difference in SRF on corresponding values (i.e., channel reflectance and spectral index) between Sentinel-2A MSI and Landsat 8 OLI was investigated. Spectra samples from ASTER Spectral Library Version 2.0 (Baldridge et al., 2009) and the Hyperion data archives (Claverie et al., 2017) were used to obtain channel reflectance through an integration process (see "2.2 Channel Reflectance").

The ASTER Spectral Library Version 2.0 contains over 2300 spectra, providing one of the most comprehensive collections of spectra covering the wavelength from the visible to thermal infrared region (Baldridge et al., 2009). The spectra in ASTER Spectral Library Version 2.0 are available from http://speclib.jpl.nasa.gov. However, as discussed previously (Chen et al., 2016), difference in wavelength range and spectral resolution between the SRFs of the channels and the spectra may result in bias in channel reflectance calculation (see Eq. (1)). Accordingly, a collection of 897 spectra obtained from ASTER Spectral Library Version 2.0 was used in further investigations. An interpolation procedure (Chen et al., 2016) was used to tackle the difference in spectral resolution.

Additionally, a collection of spectra from Hyperion data archives was selected (Claverie et al., 2017). Currently, the Hyperion spectra archive has been used for channel reflectance transformation between OLI and MSI in an initiative program called "Harmonized Landsat Sentinel-2" (Claverie et al., 2017) (also see https://hls.gsfc.nasa.gov/). The Hyperion instrument provides a high resolution hyperspectral imager which is capable of resolving 220 unique spectral channels (from $400 \mathrm{~nm}$ to $2500 \mathrm{~nm}$ ). Each channel is provided with averagely $10 \mathrm{~nm}$ full width at half maximum (FWHM) with a spatial resolution of $30 \mathrm{~m}$. The Level 1 radiometric product has a total of 242 bands, but only 198 bands are calibrated. In addition, due to an overlap between the VNIR and SWIR focal planes, there are actually only 196 unique channels. Information of all calibrated bands of Hyperion is provided publicly at https://eol.usgs.gov/sensors/hyperioncoverage. In this paper, the information of center wavelength and FWHM from the website was used to obtain the SRFs of Hyperion through an empirical model (Chen et al., 2018).

\section{RESULTS AND DISCUSSION}

The demonstration of SRFs shows difference (more or less) in channel settings between Sentinel-2A MSI and Landsat 8 OLI (Figure 1). Significant shift of SRF is observed in Blue, Green, and Red, as well as NIR (i.e., "B8", 785-900 nm), within which MSI has two channels (Figure 1 and Table 1). The difference in SRF may affect comparability between two sensors in channel reflectance and retrieved spectra index. Generally, as shown in Table 2, the RMSD of channel reflectance between MSI (Sentinel-2A) and OLI is more significant for Blue, Red, and NIR ("B8", 785-900 nm, see Figure 1 and Table 1), based on the collection of ASTER spectra (totally 897 samples). The MD shows that the channel reflectance of MSI is averagely greater than the channel reflectance of OLI over most channels. Similar findings are shown though Hyperion spectra collection separately, although individual measures are different more or less (Table 2). For the comparison of simulation based on the Hyperion spectra collection, SWIR1 channel (Table 2) was not considered mainly due to the effects of atmospheric on radiometric calibration of Hyperion.

Relative differences for all corresponding channels are demonstrated, separately based on ASTER spectra collection (Figure 2) and Hyperion spectra collection (Figure 3). Results in Figure 2 show that MSI and OLI are well comparable over several channels with small relative discrepancy (less than 5\%), including CA, 8a NIR, SWIR, and Cirrus. Meanwhile, for channels within Blue, Green, Red, and NIR (785-900 nm), samples showing significant difference (greater than $5 \%$ ) are observed obviously, most of which are with low reflectance (less than 0.5). However, findings based on Hyperion spectra collections are not completely consistent (Figure 2 and Figure 3). Significant difference is recorded in NIR (785-900 nm) for most Hyperion spectra provided with relatively low and moderate reflectance (Figure 3 ). 


\begin{tabular}{|c|c|c|c|c|c|c||}
\hline & \multicolumn{2}{|c|}{ ASTER spectra collection } & \multicolumn{3}{c|}{ Hyperion spectra collection } \\
\hline & MD & RMSD & MRD & MD & RMSD & MRD \\
\hline CA & 0.060 & 0.122 & 0.255 & -0.026 & 0.073 & -0.672 \\
\hline Blue & 0.706 & 1.144 & 2.696 & 0.840 & 0.916 & 13.588 \\
\hline Green & -0.041 & 0.261 & -0.239 & 0.041 & 0.144 & 0.770 \\
\hline Red & 0.179 & 0.409 & 0.416 & 0.228 & 0.396 & 0.945 \\
\hline NIR & 0.194 & 1.148 & 0.800 & -1.303 & 1.503 & -5.081 \\
\hline 8a NIR & -0.009 & 0.039 & -0.059 & 0.065 & 0.077 & 0.246 \\
\hline SWIR1 & -0.068 & 0.231 & -0.128 & -- & -- & -- \\
\hline SWIR2 & 0.039 & 0.137 & 0.098 & 0.148 & 0.163 & 0.720 \\
\hline Cirrus & -0.005 & 0.229 & -0.025 & 0.066 & 0.102 & 0.606 \\
\hline
\end{tabular}

Table 2. Difference measurements of channel reflectance based on the ASTER spectra collection (including 897 spectra) and the Hyperion spectra collection (including 10,000 spectra) (Note: Values of MD and RMSD in this table are scaled with 100)

Another issue related to sensor difference is the comparability in spectra index derived from channel reflectance (s). Generally, compared with the difference in reflectance of each individual channel, the difference in derived spectral indices (i.e., NDVI and NDBI) are more significant (Table 3). Instead of using the MRD, the MdRD was used to measure the average relative difference in spectral index. Comparisons of NDBI using SWIR1 derived from Hyperion spectra are not shown in Table 3 , due to atmospheric effects on radiometric calibration of Hyperion. Compared with the " 8 " NIR channel, the " $8 \mathrm{a}$ " NIR is more suitable for NDVI calculation, which improves the comparability between two sensors (Table 3 and Figure 4). Accordingly, if the effects of spatial resolution are minor or negligible, the " $8 \mathrm{a}$ " NIR is suggested for spectra indices calculation (i.e., NDVI) in terms of the cross-sensor consistency (i.e., OLI and MSI), although the " 8 " NIR is recommended officially (https://sentinel.esa.int/web/sentinel/technicalguides/ sentinel-2-msi/level-2a/algorithm).

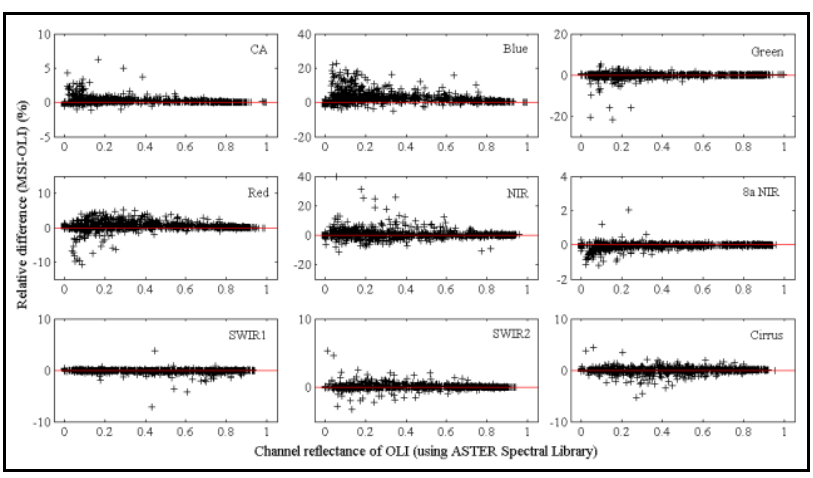

Figure 2. Relative difference in channel reflectance between MSI and OLI, based on a collection including 897 spectra from ASTER Spectral Library Version 2.0. The relative difference in this figure is defined as Eq. (6).

To improve the comparability and ensure direct comparison between observations or derived spectra indices of different sensors, linear transformation model has been used in terms of easy application (Li et al., 2014; Roy et al., 2016; Claverie et al., 2017). Improvements through linear transformation model is discussed accordingly. Due to space limitation, detailed investigations on NIR (785-900 nm) of MSI are presented in this paper. Generally, the comparability of NIR between OLI and MSI has been improved through the linear transformation. As shown in Figure 5 and Figure 6, the original difference (as mean difference) is more significant than the prediction error. Thanks to the linear transformation, the median difference in
NIR decreases to $(-0.0024 \%)$ from the original difference $(0.1916 \%)$, when ASTER spectra collection is considered (Figure 5). For Hyperion collection, the median original difference $(-1.3029 \%)$ is more obvious compared with the median difference after linear transformation (6.6218e-05\%) (Figure 6).

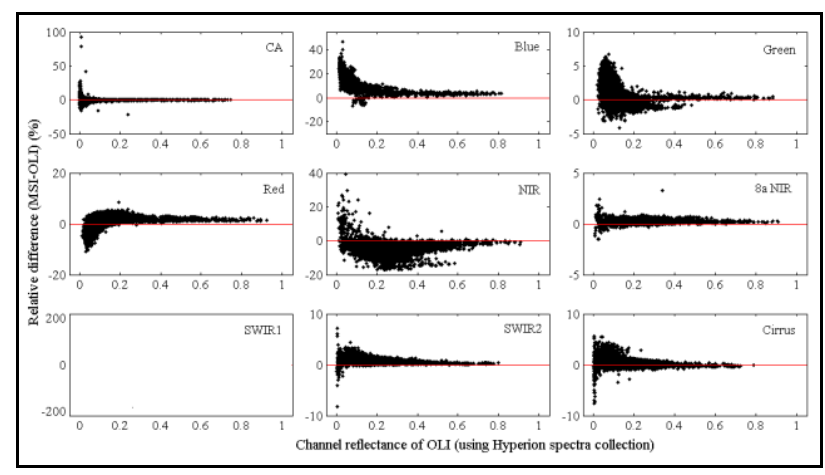

Figure 3. Same as Figure 2, but results based on samples from Hyperion spectra collection. The plot for SWIR1 is not shown due to strong impacts of atmosphere on radiometric calibration.

\begin{tabular}{||c|c|c|c|c|c|c|}
\hline & \multicolumn{3}{|c|}{ ASTER spectra collection } & \multicolumn{3}{c|}{ Hyperion spectra collection } \\
\hline & MD & RMSD & MdRD & MD & RMSD & MdRD \\
\hline NDVI & 0.176 & 1.967 & -14.866 & -2.590 & 3.172 & -9.496 \\
\hline NDVIa & -0.235 & 0.728 & -6.381 & -0.465 & 0.927 & -1.985 \\
\hline NDBI & -0.336 & 1.854 & 1.481 & -- & -- & -- \\
\hline NDBIa & 0.065 & 0.206 & 0.318 & -- & -- & -- \\
\hline
\end{tabular}

Table 3. Same as Table 2, but for two derived spectra indices (NDVI and NDBI). Both negative and positive spectra index records are included. (Index labelled with "a" is that the corresponding index for MSI is obtained by using " $8 \mathrm{a}$ " NIR)

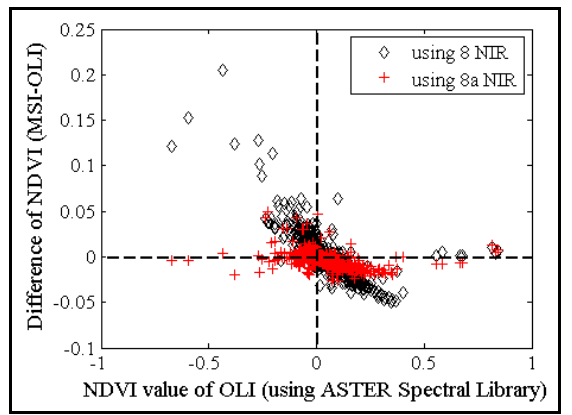

Figure 4. Difference in spectra index (NDVI) between MSI and OLI, based on a collection of 897 spectra from ASTER Spectral Library Version 2.0. For MSI, two indices are obtained using different NIR channels (labelled as " 8 " and " $8 \mathrm{a}$ ", see Table 1) respectively.

Meanwhile, variation of the model coefficients, including "Slope" and "Offset" across all K-fold cross-validation tests $(10,000$ times with $\mathrm{K}=5)$ are shown (Figure 5 and Figure 6). The "Offset" is significantly different from zero, indicating that the linear model with a constant term is reasonable for NIR (785-900 nm) transformation between MSI and OLI. Median values of "Slope" and "Offset" based on all K-fold crossvalidation cases are used to build the transformation model (Table 4). The transformation model is generally dependent on spectra collection. Differences in NIR (785-900 nm) of the transformed MSI are more significant than the original differences, if the transformation model generated from another spectra collection is used. For example, if M1 (Table 4) is 
applied to transform NIR of Hyperion spectra collection, the discrepancy between transformed MSI and OLI generally increases (Table 5). That is to say, improvement in betweensensor consistency is possibly a challenge, through linear transformation based on model (s) generated from other different spectra collections.

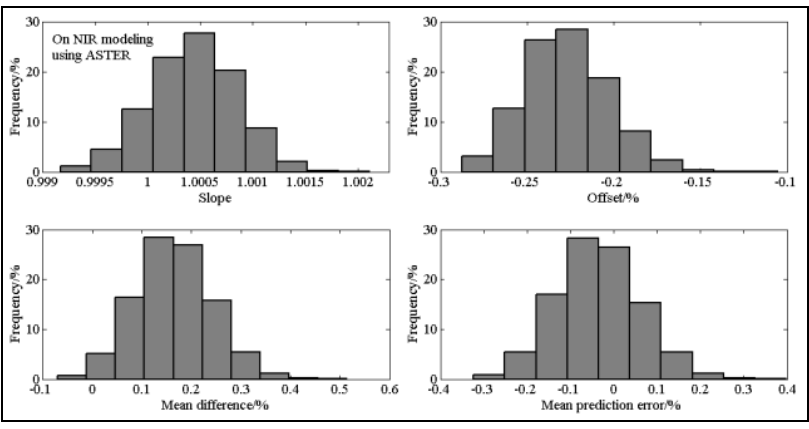

Figure 5. Uncertainty of the linear transformation model for NIR (785-900 nm), based on the ASTER spectra collection. In this figure, "Mean difference" is the original difference between testing pairs without/before transformation and "Mean prediction error" is the difference after modelling transformation. The statistics are based on 10,000 times K-fold cross-validation $(\mathrm{K}=5)$.

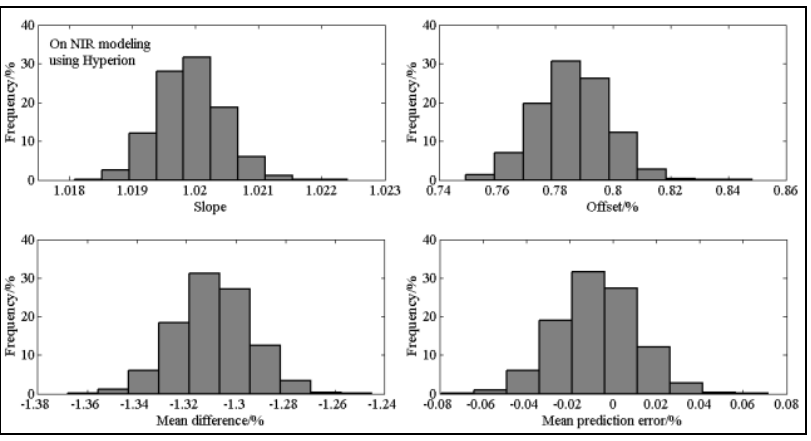

Figure 6. Same as Figure 5, but the statistics of modelling results are based on Hyperion spectra collection.

\begin{tabular}{|c|c|}
\hline Spectra collection & Linear transformation model for NIR \\
\hline ASTER & OLI $=1.0006 \times$ MSI $-0.2193 \mathrm{e}-2(\mathrm{M} 1)$ \\
\hline Hyperion & OLI $=1.0201 \times$ MSI $+0.7910 \mathrm{e}-2(\mathrm{M} 2)$ \\
\hline
\end{tabular}

Table 4. Linear transformation model for NIR channel (785$900 \mathrm{~nm}$ ) using the median coefficients in Figure 5 and Figure 6.

\begin{tabular}{|c|c|c|}
\hline Spectra collection & Model based on ASTER & Model based on Hyperion \\
\hline ASTER & $-1.5601 \mathrm{e}-05$ & 1.85 \\
\hline Hyperion & -1.51 & $-5.840 \mathrm{e}-04$ \\
\hline
\end{tabular}

Table 5. Mean difference (MD) scaled with 100 in NIR (785$900 \mathrm{~nm}$ ) reflectance between OLI and transformed MSI using corresponding model presented in Table 4.

\section{CONCLUSIONS}

In conclusion, the preliminary investigation shows general results of the comparison of MSI (mainly on Sentinel-2A) and Landsat 8 OLI in terms of corresponding values (e.g., channel reflectance and several derived spectral indices, including
NDVI and NDBI). Findings in this paper are mainly based on simulations separately from a spectra collection of ASTER Spectral Library Version 2.0 and a Hyperion spectra collection with 10,000 samples. Generally, the difference is more significant for Blue, Red, and "8" NIR (785-900 nm). Compared with the difference in reflectance of each individual channel, the difference in derived spectral indices (i.e., NDVI and NDBI) are more significant. For MSI, compared with the " 8 " NIR (785-900 nm) channel, the " $8 \mathrm{a}$ " NIR $(855-875 \mathrm{~nm})$ is more applicable for NDVI calculation, in terms of decreasing the between-sensor difference.

Linear transformation model widely used previously for sensors cross-calibration is a practical way to improve between-sensor consistency. However, the improvements are sensitive to transformation model. If an improper transformation model is selected, the between-sensor discrepancy will even largely increase. Improvement in between-sensor consistency is possibly a challenge, through linear transformation model (s) generated from other different spectra collections. Actually, challenges in making consistency among the Landsat series are also there, which need to be tackled well to fully use the observations by Sentinel-2 and Landsat series.

\section{ACKNOWLEDGEMENTS}

This research was jointly supported by China Postdoctoral Science Foundation (Grant 2017M612124), the National Key Research and Development Program of China (Grant 2016YFC1401008 and 2016YFC1401001). Appreciations are given to Dr. Simon Hook at NASA JPL, for the provision of ASTER Spectral Library Version 2.0. We acknowledge Prof. Su Z. (Bob) of University of Twente and Prof. Qihao Weng of Indiana State University, for their valuable suggestions during this investigation.

\section{REFERENCES}

Baldridge, A.M., Hook, S.J., Grove, C.I., and Rivera, G., 2009. The ASTER spectral library version 2.0. Remote Sens. Environ., 113, pp. 711-715.

Claverie, M., Masek, J.G., Ju, J., and Dungan, J.L., 2017. Harmonized Landsat-8 Sentinel-2 (HLS) Product User's Guide. https://hls.gsfc.nasa.gov/documents/.

Chander, G., Helder, D.L., Aaron, D.B., Mishra, N., and Shrestha, A.K., 2013. Assessment of spectral, misregistration, and spatial uncertainties inherent in the cross-calibration study. IEEE T. Geosci. Remote., 51(3), pp.1282-1296.

Chen, F., Yang, S., Su, Z., and Wang, K., 2016. Effect of emissivity uncertainty on surface temperature retrieval over urban areas: Investigations based on spectral libraries. ISPRS J. Photogramm., 114, pp. 53-65.

Chen, F., Yang, S., Yin, K., and Chan, P., 2017. Challenges to quantitative applications of Landsat observations for the urban thermal environment. J. Environ. Sci.-China, 59, 80-88.

Chen, F., Li, J., Wang, C., and Claverie, M., 2018. A comparison of Sentinel-2A and Sentinel-2B with preliminary results. International Geoscience and Remote Sensing Symposium, Valencia, Spain. (Accepted) 
Li, P., Jiang, L., and Feng, Z., 2014. Cross-comparison of vegetation indices derived from Landsat-7 Enhanced Thematic Mapper Plus (ETM+) and Landsat-8 Operational Land Imager (OLI) sensors. Remote Sens.-Basel, 6(1), 310-329.

Mandanici, E., and Bitelli, G., 2016. Preliminary comparison of Sentinel-2 and Landsat 8 imagery for a combined use. Remote Sens.-Basel, 8, pp.1014.

Roy, D.P., Kovalskyy, V., Zhang, H.K., Vermote, E.F., Yan, L., Kumar, S.S., and Egorov, A., 2016. Characterization of Landsat-7 to Landsat-8 reflective wavelength and normalized difference vegetation index continuity. Remote Sens. Environ., 185 , pp. $57-70$

Xiong, Y.Z., Huang, S.P., Chen, F., Ye, H., Wang, C.P., and Zhu, C.B., 2012. The impacts of rapid urbanization on the thermal environment: a remote sensing study of Guangzhou, South China. Remote Sens.-Basel, 4(7), 2033-2056.

Zha, Y., Gao, J., and Ni, S., 2003. Use of normalized difference built-up index in automatically mapping urban areas from TM imagery. Int. J. Remote Sens., 24, pp. 583-594. 